\title{
Endoloop application as an alternative method for gastrotomy closure in experimental transgastric surgery
}

\author{
D. Katsarelias · Andreas Polydorou - Athanasia Tsaroucha • \\ Emmanuel Pavlakis · Georgia Dedemadi · Leda Pistiolis · \\ Nikolaos Karakostas · Agatha Kondi-Paphiti · Elias Mallas
}

Published online: 18 August 2007

(C) Springer Science+Business Media, LLC 2007

\section{Erratum to: Surg Endosc}

\section{DOI 10.1007/s00464-007-9281-1}

The following coauthors, also affiliated with Aretaieion Hospital in Athens, Greece, were inadvertently omitted:

Andreas Polydorou

Athanasia Tsaroucha

Emmanuel Pavlakis

Georgia Dedemadi

Leda Pistiolis

Nikolaos Karakostas

Agatha Kondi-Paphiti

Elias Mallas

The online version of the original article can be found under doi:10.1007/s00464-007-9281-1

D. Katsarelias $(\bowtie) \cdot$ A. Polydorou · A. Tsaroucha .

E. Pavlakis · G. Dedemadi · L. Pistiolis · N. Karakostas ·

A. Kondi-Paphiti · E. Mallas

Department of Surgery, Aretaieion Hospital,

V. Sofias 76 avenue, 11527 Athens, Greece

e-mail: dkatsarelias@gmail.com 